\title{
Cloud Ablation by a Relativistic Jet and the Extended Flare in CTA 102 in 2016 and 2017
}

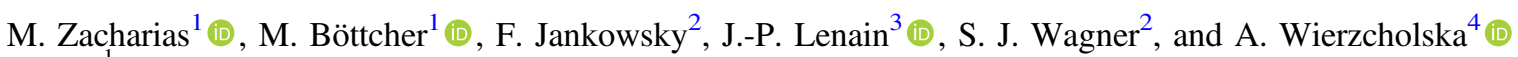 \\ ${ }^{1}$ Centre for Space Science, North-West University, Potchefstroom, 2520, South Africa; mzacharias.phys@gmail.com \\ ${ }^{2}$ Landessternwarte, Universität Heidelberg, Königstuhl, D-69117 Heidelberg, Germany \\ ${ }^{3}$ Sorbonne Universités, UPMC Université Paris 06, Université Paris Diderot, Sorbonne Paris Cité, CNRS, Laboratoire de Physique Nucléaire et de Hautes Energies \\ (LPNHE), 4 place Jussieu, F-75252, Paris Cedex 5, France \\ ${ }^{4}$ Institute of Nuclear Physics, Polish Academy of Sciences, PL-31342 Krakow, Poland \\ Received 2017 September 29; revised 2017 November 15; accepted 2017 November 16; published 2017 December 14
}

\begin{abstract}
In late 2016 and early 2017, the flat spectrum radio quasar CTA 102 exhibited a very strong and long-lasting outburst. The event can be described by a roughly two-month long increase of the baseline flux in the monitored energy bands (optical to $\gamma$-rays) by a factor 8 , and a subsequent decrease over another two months back to pre-flare levels. The long-term trend was superseded by short but very strong flares, resulting in a peak flux that was a factor 50 above pre-flare levels in the $\gamma$-ray domain and almost a factor 100 above pre-flare levels in the optical domain. In this paper, we explain the long-term evolution of the outburst by the ablation of a gas cloud penetrating the relativistic jet. The slice-by-slice ablation results in a gradual increase of the particle injection until the center of the cloud is reached, after which the injected number of particles decreases again. With reasonable cloud parameters, we obtain excellent fits of the long-term trend.
\end{abstract}

Key words: galaxies: active - quasars: individual (CTA 102) - radiation mechanisms: non-thermal - relativistic processes

\section{Introduction}

Blazars, the relativistically beamed, radio-loud version of active galactic nuclei (Blandford \& Rees 1974), are historically categorized into two classes depending on the width of their optical emission lines: BL Lacertae objects with line equivalent width $\mathrm{EW}<5 \AA$, and flat spectrum radio quasars (FSRQs) with $\mathrm{EW}>5 \AA$. The latter case indicates the presence of a strong broad-line region (BLR) surrounding the central supermassive black hole on scales of $\sim 0.1 \mathrm{pc}$. The origin of the double-humped spectral energy distribution (SED) is regarded by most authors to be synchrotron and inverse-Compton (IC) emission of particles within the relativistic jet, with electrons and positrons being responsible for the emission, and protons serving as a cold background. Especially in FSRQs, seed photon fields for the IC process are abundant. Apart from the emission region's internal synchrotron emission (resulting in synchrotron self-Compton, SSC, flux), the external fields from the accretion disk, the BLR, or the dusty torus are also potential targets depending on the distance of the emission region from the black hole.

Blazars are strongly variable in all energy bands. The large variety in flaring events has led to a similarly large number of models. A particularly interesting case is the interaction of the jet with an obstacle, such as a star (Blandford \& Königl 1979; Komissarov 1994; Perucho et al. 2014; Bosch-Ramon 2015), its wind (Araudo et al. 2009; de la Cita et al. 2017), or a gas cloud (Araudo et al. 2010; Bosch-Ramon et al. 2012). What most of these models have in common is that the obstacle is already fully inside the jet before the start of the interaction. However, given the strong pressure of the relativistically moving matter of the jet, interactions will start as soon as the obstacle hits the jet, since the jet will look like a strong shock. Simulations of shock/cloud interactions have shown that a cloud will be quickly ripped apart (Klein et al. 1994; Poludnenko et al. 2002). Recent simulations of a jet/cloud (Bosch-Ramon et al. 2012) or jet/star (Perucho et al. 2017) interaction, where the penetration process is included, reveal that the obstacle is (partially) ablated, and a significant amount of matter is mixed into the jet flow.

This is easy to see for a gas cloud, given that it is mainly confined by its own, rather weak gravity. The ram pressure of the jet will immediately start to ablate the outer layers of the cloud while it starts to penetrate the jet. The mass loss of the cloud will weaken its structural integrity even before it has fully penetrated the jet. As we will discuss below, the cloud will be ablated and carried along by the jet. Depending on the cloud parameters, such as size and velocity, this might lead to pronounced and prolonged jet activity, when the additional material in the jet reaches an internal shock located downstream of the cloud penetration site. We apply this model to a recent flare in CTA 102, where fluxes varied significantly over several months.

CTA 102 is an FSRQ at a redshift $z_{\text {red }}=1.037$, roughly halfway across the observable universe. The accretion disk luminosity is $L_{\text {disk }}^{\prime}=3.8 \times 10^{46} \mathrm{erg} \mathrm{s}^{-1}$ (Zamaninasab et al. 2014). The mass of the central black hole is estimated at $M_{\mathrm{bh}} \sim 8.5 \times 10^{8} M_{\odot}$ (Zamaninasab et al. 2014), giving an Eddington luminosity of $L_{\text {Edd }}^{\prime} \sim 1.1 \times 10^{47} \mathrm{erg} \mathrm{s}^{-1}$. The BLR properties were derived by Pian et al. (2005) using UV spectroscopy observations with the Hubble Space Telescope, resulting in a luminosity of $L_{\mathrm{BLR}}^{\prime}=4.14 \times 10^{45} \mathrm{erg} \mathrm{s}^{-1}$ and a radius of $R_{\mathrm{BLR}}^{\prime}=6.7 \times 10^{17} \mathrm{~cm}$ (all quantities given in the AGN frame).

Long-term observations in radio bands since 1980 (Fromm et al. 2011) revealed a rather dormant source until $~ 1997$, after which it showed a few radio outbursts with a particularly strong one in 2006. Fromm et al. (2011) favor a shock-shock interaction scenario to explain the observed evolution of the latter event. Similarly, in the high-energy (HE; $E>100 \mathrm{MeV}$ ) $\gamma$-ray band, scanned continuously by the Fermi satellite since mid-2008, CTA 102 showed low fluxes in the first almost four years of Fermi-LAT operation with an average flux above 
$1 \mathrm{GeV}$ of $(5.0 \pm 0.2) \times 10^{-9} \mathrm{ph} \mathrm{cm}^{-2} \mathrm{~s}^{-1}$ and a photon index of $\Gamma=2.34 \pm 0.03$ (Acero et al. 2015). In the second half of 2012, CTA 102 exhibited a strong $\gamma$-ray outburst with a peak flux above $100 \mathrm{MeV}$ of $\sim 8 \times 10^{-6} \mathrm{ph} \mathrm{cm}^{-2} \mathrm{~s}^{-1}$. This outburst along with the correlated optical variability led Larionov et al. (2016) to propose the helical motion and the accompanied variation of the Doppler factor of a plasma blob (Schramm et al. 1993) as the main driver of the flare. Since 2012, CTA 102 has remained active without long returns to pre-flare levels in both the $\gamma$-ray and optical bands. However, all of these outbursts have been rather short-lived on the order of a few days, with fast rises to the maximum and subsequent quick decays.

This behavior changed in late 2016, when CTA 102 entered into a prolonged activity phase, which saw both the $\gamma$-ray and optical fluxes, as well as the X-ray flux, rising continuously for about two months. The peak fluxes were obtained at the end of 2016 December, which were in all cases significantly higher than any previously observed fluxes. The optical fluxes exhibited clear intranight variability (Bachev et al. 2017). Subsequently, the $\gamma$-ray flux decreased over the course of about two months to 2016 October levels. Unfortunately, this decrease of flux could not be observed in optical or X-ray observations due to Sun constraints.

In this paper, we present the multiwavelength data of this roughly four-month-long outburst and explain it by the ablation of a gas cloud by the relativistic jet. The initial density increase in ablated material causes the rise of the light curve, while the ablation of the second half of the cloud exhibits a decrease in ablated material, resulting in the subsequent drop of the light curve. Our focus is on the explanation of the long-term trend, and we do not deal with the fast variability on top of the longer trend. The paper is organized as follows. First, we present the data analysis in Section 2. Section 3 describes the theoretical model of cloud ablation, followed by a summary of the code used and the modeling in Section 4. We discuss and conclude in Section 5.

In the following sections, primed quantities are in the AGN frame, quantities marked with the superscript "obs" are in the observer's frame, and unmarked quantities are in the comoving jet frame. We use a standard, flat cosmology with $H_{0}=69.6 \mathrm{~km} \mathrm{~s}^{-1} \mathrm{Mpc}^{-1}$ and $\Omega_{M}=0.27$, which gives a luminosity distance $d_{L}=2.19 \times 10^{28} \mathrm{~cm}$.

\section{Data Analysis}

The flare in CTA 102 was extensively observed by a large number of observatories. Here, we analyze and report the detailed observations of Fermi-LAT in the $\gamma$-ray band, SwiftX-ray Telescope (XRT) in the X-ray band, as well as the SwiftUVOT and Automatic Telescope for Optical Monitoring (ATOM) in the optical band.

\subsection{Fermi-LAT Data Analysis}

The LAT instrument (Atwood et al. 2009) on board the Fermi satellite surveys the high-energy $\gamma$-ray sky every $3 \mathrm{hr}$, with energies between $20 \mathrm{MeV}$ and above $300 \mathrm{GeV}$, thus making it an ideal instrument to monitor the activity of CTA 102. This AGN has been reported in all of the available FermiLAT catalogs and is identified as 3FGL J2232.5+1143 in the third Fermi-LAT source catalog (Acero et al. 2015).

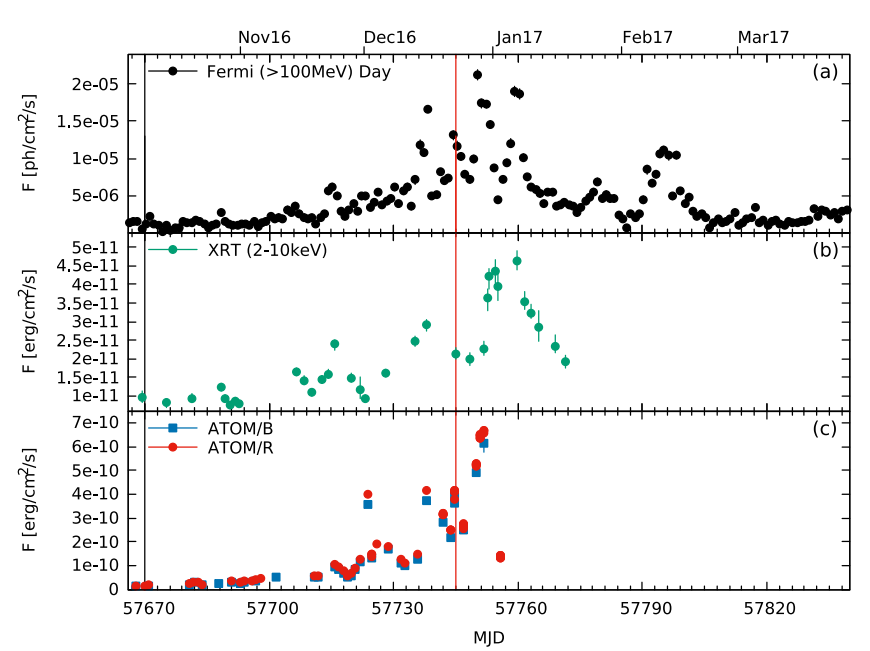

Figure 1. Light curves of (a) Fermi-LAT data, (b) Swift-XRT data, and (c) optical data from ATOM as labeled. The vertical thin black and red lines mark the dates when the spectra were extracted.

The Fermi-LAT data are analyzed using the public ScienceTools v10r0p5..$^{5}$ Events in a circular region of interest of $10^{\circ}$ in radius are extracted, centered on the nominal position of 3FGL J2232.5+1143. To probe the active state reported here, only data between 2015 August 8 (MJD 57235) and 2017 May 1 (MJD 57874), in the $100 \mathrm{MeV}-500 \mathrm{GeV}$ energy range, are considered. The P8R2_SOURCE_V6 instrument response functions (event class 128 and event type 3) were used, together with a zenith angle cut of $90^{\circ}$ to avoid contamination by the $\gamma$-ray-bright Earth-limb emission. The model of the region of interest was built based on the 3FGL catalog (Acero et al. 2015). The Galactic diffuse emission was modeled using the file gll_iem_v06.fits (Acero et al. 2016) and the isotropic background using iso_P8R2_SOURCE_V6_V06.txt. In the following, the source spectrum will be investigated both with a power-law shape,

$$
\frac{d N}{d E}=N_{0}\left(\frac{E}{E_{0}}\right)^{-\Gamma},
$$

and a log-parabola,

$$
\frac{d N}{d E}=N_{0}\left(\frac{E}{E_{b}}\right)^{-\left(\Gamma+\beta \log \left(E / E_{b}\right)\right)}
$$

with $E_{b}=308 \mathrm{MeV}$ fixed to the value reported in the 3FGL catalog.

For the considered period between 2015 August and 2017 May, CTA 102 is detected with a Test Statistic (TS; Mattox et al. 1996) of 163,879, i.e., $\sim 405 \sigma$. The spectrum of CTA 102 is significantly curved with a photon index of $\Gamma=2.068 \pm$ 0.008 and a curvature index of $\beta=0.064 \pm 0.003$. The average flux is $F=(2.27 \pm 0.01) \times 10^{-6} \mathrm{ph} \mathrm{cm}^{-2} \mathrm{~s}^{-1}$.

To further study the activity of CTA 102 at high energies, a light curve has been produced with a time binning of 1 day. Since on these timescales the preference for a log-parabola is not guaranteed, the spectrum has been modeled with a simple power law in each time bin, leaving the photon index free to vary. The resulting light curve is shown in Figure 1(a).

From this data set, spectra were derived for two particular dates: MJD 57670 and MJD 57745, which are representative of

\footnotetext{
See http://fermi.gsfc.nasa.gov/ssc/data/analysis/documentation.
} 
the pre-flare state and the flare state around the maximum. For MJD 57670, CTA 102 is detected with TS $=161(\sim 12 \sigma)$, and the observed spectrum is well-described by a power law with $F=(1.19 \pm 0.23) \times 10^{-6} \mathrm{ph} \mathrm{cm}^{-2} \mathrm{~s}^{-1}$ and $\Gamma=2.08 \pm 0.14$. Testing a log-parabola only yielded a log-likelihood ratio 0.2 with respect to a power law. In order to validate that the nondetection of curvature is independent of the detection significance, we derived a 10 day spectrum starting on MJD 57670. Despite the increased significance of the source with TS $=683(\sim 26 \sigma)$, the spectrum is still compatible with a power law, since the log-parabola is only preferred with $0.95 \sigma$. For MJD 57745, the detection level of CTA 102 reaches $\mathrm{TS}=4558(\sim 67 \sigma)$, and the observed source spectrum is significantly curved, with a log-likelihood ratio of 9.9 for a logparabolic spectrum with respect to a power law. The corresponding spectrum results in $F=(1.10 \pm 0.05) \times$ $10^{-5} \mathrm{ph} \mathrm{cm}^{-2} \mathrm{~s}^{-1}, \quad \Gamma=1.797 \pm 0.061$ and $\beta=0.077 \pm$ 0.025 . The two one-day spectra are shown in Figure 4 as the black and red butterflies, respectively. The displayed spectra were corrected for absorption by the extragalactic background light (EBL) following the model of Franceschini et al. (2008), which has, however, only a minor influence at the highest energies.

The change in spectral shape can be interpreted as a move of the peak energy during the flare toward higher energies. Although the peak of the IC component cannot be determined before the flare (somewhere between $10 \mathrm{keV}$ and $100 \mathrm{MeV}$ ), during the peak of the flare it is at about $3 \mathrm{GeV}$. This points toward a significant hardening of the underlying particle distribution.

\subsection{X-Ray Analysis}

The Swift Gamma-Ray Burst Mission (hereafter Swift; Gehrels et al. 2004) is a multifrequency space observatory that allows targets in the optical, ultraviolet, and X-ray energy bands to be monitored. The XRT (Burrows et al. 2005) has monitored CTA 102 since 2005 in 137 pointing observations taken in the energy range of $0.3-10 \mathrm{keV}$. In this work, the light curve (Figure 1(b)) presents data collected between MJD 57668 and MJD 57821, which correspond to the ObsIDs of 00033509084-00033509120.

All data collected were analyzed using version 6.20 of the HEASOFT package. ${ }^{6}$ The data were recalibrated using the standard procedure xrtpipeline. For the spectral fitting, XSPEC v.12.8.2 was used (Arnaud 1996). All data were binned to have at least 30 counts per bin. Each observation was fitted using the power-law model, Equation (1), with the Galactic absorption value of $N_{H}=4.76 \times 10^{20} \mathrm{~cm}^{-2}$ (Kalberla et al. 2005) set as a frozen parameter. In each observation, we also checked if a broken power-law model can result in a better description of the spectrum. According to reduced $\chi^{2}$ values, a simple power law is the best model for all data in our set.

The two observations presented in the global SED (Figure 4) are described with the following spectral parameters: $\Gamma_{57670}=$ $1.3 \pm 0.2$ and $N_{57670}=(1.17 \pm 0.16) \times 10^{-3} \mathrm{~cm}^{-2} \mathrm{~s}^{-1} \mathrm{keV}^{-1}$ and $\Gamma_{57745}=1.52 \pm 0.06$ and $N_{57745}=(3.93 \pm 0.18) \times$ $10^{-3} \mathrm{~cm}^{-2} \mathrm{~s}^{-1} \mathrm{keV}^{-1}$. The spectrum shown with black symbols corresponds to observations taken nearest to MJD 57670, which is data with ObsId 00033509084, while red symbols

\footnotetext{
6 http://heasarc.gsfc.nasa.gov/docs/software/lheasoft
}

correspond to the observations taken nearest to MJD 57745, which is data with ObsId 00033509109. Apparently, only the normalization of the spectra changes.

\subsection{Optical/UV Analysis}

Simultaneously with XRT, CTA 102 was monitored with the UVOT instrument on board Swift. The observations were taken in the UV and optical bands with the central wavelengths of $U V W 2(188 \mathrm{~nm}), U V M 2(217 \mathrm{~nm}), U V W 1(251 \mathrm{~nm})$, $\mathrm{U}(345 \mathrm{~nm}), B(439 \mathrm{~nm})$, and $V(544 \mathrm{~nm})$. The instrumental magnitudes were calculated using the uvotsource task including all photons from a circular region with radius $5^{\prime \prime}$. The background was determined from a circular region with a radius of $5^{\prime \prime}$ near the source region that is not contaminated with signal from any nearby source. The optical and ultraviolet data points were corrected for dust absorption using the reddening $E(B-V)=0.0612 \mathrm{mag}$ (Schlafly \& Finkbeiner 2011) and the ratios of the extinction to reddening, $A_{\lambda} / E(B-V)$ (Giommi et al. 2006).

Further optical data in the $R$ - and $B$-band filters were obtained with the ATOM, which is a $75 \mathrm{~cm}$ optical telescope located at the H.E.S.S. site in the Khomas Highland in Namibia (Hauser et al. 2004). It regularly observes roughly $250 \gamma$-ray emitters.

ATOM has monitored CTA 102 since 2008. During the visibility period presented in this paper, $R$-band monitoring lasted from 2016 June until 2017 January. Additional $B$-band observations were taken from 2016 October until 2016 December. Most of the high-flux period is covered by at least one $B$-band and several $R$-band measurements per night. The data were analyzed using the fully automated ATOM Data Reduction and Analysis Software and were manually quality checked. The resulting flux was calculated via differential photometry using five custom-calibrated secondary standard stars in the same field of view.

Using measurements from a calm period between 2008 and 2011, the baseline flux of CTA 102 can be established as $R=16.90 \pm 0.02 \mathrm{mag}$. An outburst in 2012 September reached $R=14.6 \pm 0.1 \mathrm{mag}$ before returning to previous levels. In late 2015, ATOM detected CTA 102 at $R=16.54 \pm 0.08$ mag. Beginning in mid-2016, CTA 102 showed increasing activity, with a first outburst in August reaching $R=14.20 \pm 0.02 \mathrm{mag}$. Toward the end of visibility, CTA 102 started to steadily brighten, culminating in $R=10.96 \pm 0.05 \mathrm{mag}$ on 2016 December 29 (MJD 57751). We find significant intranight variability, similar to the results reported in Bachev et al. (2017). Both $R$ - and $B$-band light curves are shown in Figure 1(c).

We confirmed that the color of the optical/UV spectra is constant in time, which implies that the peak of the synchrotron component does not move significantly from its initial, unknown position in the infrared toward bluer, optical frequencies. This has the unfortunate side effect that we cannot determine the peak synchrotron energy during this flare. On the other hand, one can deduce that neither the maximum Lorentz factor of the electrons nor the magnetic field increases significantly.

\subsection{Flux Evolution After 2017 March}

Between mid-January and late April, the source is not visible to optical and X-ray observatories, since CTA 102 is too close 
to the Sun during these months. Hence, the downward trend visible in the $\gamma$-ray light curve could not be observed in any other band. Swift and ATOM resumed observations of CTA 102 in late April.

The optical flux was still highly variable between $R=16 \mathrm{mag}$ and $R=13 \mathrm{mag}$ while displaying a general trend of becoming faint. The behavior in the X-ray band was similar. In the $\gamma$-ray domain, fluxes became variable again in early April, exhibiting day-long outbursts similar to the behavior before 2016 October. Therefore, we conclude that the optical and X-ray activity at that time is unrelated to the $\gamma$-ray activity between 2016 October and 2017 March and of no concern for our modeling.

\section{Cloud Ablation by the Relativistic Jet}

The potential cause of a strong outburst is the accumulation of more matter than usual in a standing shock within the jet. If a gas cloud on its orbit around the black hole happens to penetrate the jet, it will be ablated and carried along by the jet. Hence, this is an efficient process for the jet to pick up a large amount of material and to cause prolonged jet activity, if the cloud is ablated at a steady pace while it enters the jet. Given the changing density within the cloud, the jet ablates different amounts of matter at a given time while the cloud penetrates the jet. This leads to a gradual increase and decrease of the light curve during the flare over the timescale the cloud is ablated. Density fluctuations within the cloud and instabilities during the process might lead to a more chaotic ablation, which could result in strong and fast fluctuations related to the size of these fluctuations on top of the longer trend. In the following, we will concentrate on the long-term trend and discuss the influence of density fluctuations elsewhere.

The situation is that a spherical cloud approaches the jet with orbital speed around the central black hole

$$
v_{c}^{\prime}=\sqrt{G M_{\mathrm{bh}} / z^{\prime}}
$$

where $G$ is the gravitational constant and $z^{\prime}$ the distance between the cloud and the black hole. The radius of the cloud can be derived from the rising time $t_{f}^{\prime}$ (that is, from the beginning to the peak) of the event:

$$
R_{c}^{\prime}=t_{f}^{\prime} v_{c}^{\prime}=\frac{t_{f}^{\mathrm{obs}} v_{c}^{\prime}}{\left(1+z_{\mathrm{red}}\right)}
$$

Apart from the redshift correction, the frame of the cloud and the observer's frame are identical, since the motion of the cloud is nonrelativistic. Hence, the observed duration of the flare is indeed the same as the cloud penetration time.

The number of particles in the cloud follows from the increase in particles in the jet, under the assumption that the cloud is fully ablated. We can calculate the particles in the cloud if we take the difference between the particles at the peak and at the beginning of the flare. This includes the simplifying assumption that the cloud contains a pure hydrogen plasma. Within the emission region of the jet, the density of electrons (and possibly positrons) is $n_{j, e}$. The electron charge is balanced by a fraction $a \leqslant 1$ of protons, depending on the number of positrons in the jet. The total density of particles in the jet is $n_{j}=(1+a) n_{\mathrm{j}, \mathrm{e}}$. The number of particles in the emission region obviously is $N_{j}=\frac{4}{3} \pi R_{j}^{3} n_{j}$, which is an invariant quantity. $R_{j}$ is the radius of the jet. Hence, the number of particles in the cloud equals the difference in the particle number in the jet at the peak of the event to the beginning of the event:

$$
\begin{aligned}
N_{c}^{\prime} & =N_{c}=2\left(N_{\mathrm{j}, \max }-N_{\mathrm{j}, \text { min }}\right) \\
& =\frac{8 \pi}{3} R_{j}^{3}(1+a)(U-1) n_{j, e, \text { min }} .
\end{aligned}
$$

The factor 2 takes into account that the maximum of the event takes place when the center of the cloud is ablated and the second half of the cloud is still to be ablated. $U=n_{j, e, \max } / n_{j, e, \min }$ marks the ratio of the electron densities at the peak and the beginning of the flare. Naturally, in the cloud, $a=1$. Hence, the addition of cloud material into the jet should raise the value of $a$ in the jet emission region. For ease of computation, we ignore this effect here. For an initial jet plasma with $a \lesssim 1$, the influence is negligible.

The jet ablates the cloud due to its ram pressure, which is in the black hole frame,

$$
\begin{aligned}
p_{\text {ram }}^{\prime} & =\left(\Gamma_{j}-1\right) n_{j, e, \min }^{\prime} \bar{\gamma}_{e} m_{e} c^{2}+\left(\Gamma_{j}-1\right) n_{j, p, \min }^{\prime} m_{p} c^{2} \\
& =\Gamma_{j}\left(\Gamma_{j}-1\right) \bar{\gamma}_{e} m_{e} c^{2}\left(1+a \frac{m_{p}}{\bar{\gamma}_{e} m_{e}}\right) n_{j, e, \min },
\end{aligned}
$$

introducing the bulk Lorentz factor $\Gamma_{j}$ of the jet and the speed of light $c$. Not surprisingly, for fractions of protons $1 \geqslant a>\bar{\gamma}_{e} m_{e} / m_{p}$, with the average electron Lorentz factor $\bar{\gamma}_{e}$, the electron mass $m_{e}$, and the proton mass $m_{p}$, the ram pressure is dominated by protons. Since the ram pressure of the jet is provided by particles already present in the jet, it remains constant throughout the flare and can be reconstructed by preflare parameters.

The gravitational pressure that keeps the cloud together is

$$
p_{g}^{\prime}\left(r_{c}\right)=\frac{F_{g}^{\prime}\left(r_{c}^{\prime}\right)}{A_{H}}=\frac{G M_{c}\left(r_{c}^{\prime}\right) m_{H}}{\pi r_{H}^{2} r_{c}^{\prime 2}}
$$

where $A_{H}=\pi r_{H}^{2} \sim 8.8 \times 10^{-17} \mathrm{~cm}^{2}$ is the cross-section and $m_{H} \sim m_{p}$ is the mass of a hydrogen atom, which constitutes the bulk of the particles in the cloud. $M_{c}\left(r_{c}^{\prime}\right)$ is the enclosed mass at cloud radius $r_{c}^{\prime}$.

The cloud will be ablated if $p_{\text {ram }}^{\prime}>p_{g}^{\prime}$. Hence, with Equations (6) and (7), and a slight redistribution, we can construct a lower limit on the initial jet electron density:

$$
n_{j, e, \min }>\frac{G m_{H}}{\pi r_{H}^{2} m_{e} c^{2}} \frac{M_{c}\left(r_{c}^{\prime}\right)}{\Gamma_{j}\left(\Gamma_{j}-1\right) \bar{\gamma}_{e}\left(1+a \frac{m_{p}}{\bar{\gamma}_{e} m_{e}}\right) r_{c}^{\prime 2}} .
$$

In order to get an estimate on the required jet electron density, we chose the outer layer of the cloud $r_{c}^{\prime}=R_{c}^{\prime}$ as an example. Approximating $\bar{\gamma}_{e} \ll m_{p} / m_{e}$ and $m_{H} \sim m_{p}$, we find

$$
\begin{aligned}
n_{j, e, \min } \gtrsim & 2.8 \times 10^{-12}\left(\frac{a}{0.1}\right)^{-1}\left(\frac{\Gamma_{j}}{10}\right)^{-1}\left(\frac{\Gamma_{j}-1}{9}\right)^{-1} \\
& \times\left(\frac{M_{c}}{0.01 M_{\odot}}\right)\left(\frac{R_{c}^{\prime}}{10^{15} \mathrm{~cm}}\right)^{-2} \mathrm{~cm}^{-3} .
\end{aligned}
$$



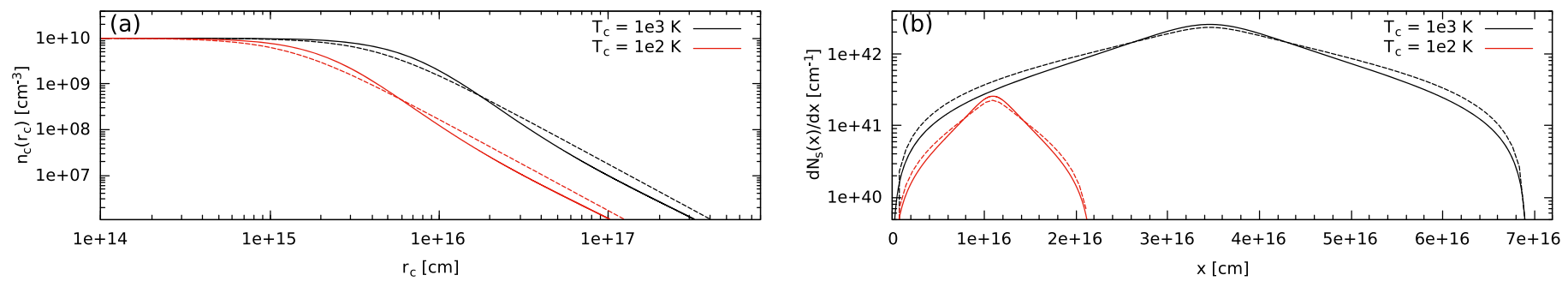

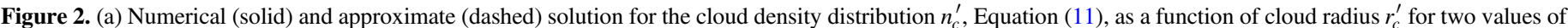

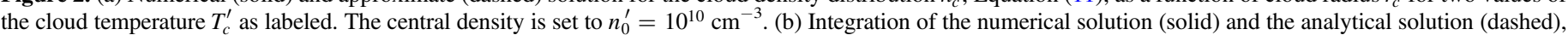

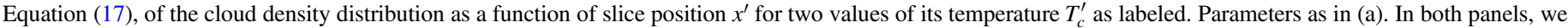
dropped the primes for clarity.

Obviously, the cloud cannot withstand destruction. Even a solar-like star with much higher surface gravity could be stripped of its outer layers while penetrating the jet, which typically exhibits electron densities exceeding $10^{-2} \mathrm{~cm}^{-3}$. However, this estimate might not hold for the inner, dense core of a star.

Given that the cloud penetrates the jet gradually, the number of particles injected into the jet changes over time. In order to calculate the correct injection term, the density distribution of the cloud $n_{c}^{\prime}\left(r_{c}^{\prime}\right)$ must be known. We consider a profile based on hydrostatic equilibrium. The simplest ansatz would be to assume that the cloud consists of an isothermal ideal gas with temperature $T_{c}^{\prime}$, so that the thermal pressure $p_{T}^{\prime}=\rho_{c}^{\prime} k_{B} T_{c}^{\prime} / m_{p}$, where $\rho_{c}^{\prime}=m_{p} n_{c}^{\prime}$ is the cloud's mass density, and $k_{B}$ is the Boltzmann constant. In this case, the equation of hydrostatic equilibrium reads

$$
\begin{aligned}
\frac{k_{B} T_{c}^{\prime}}{m_{p}} \frac{d \rho_{c}^{\prime}\left(r_{c}^{\prime}\right)}{d r_{c}^{\prime}} & =-g\left(r_{c}^{\prime}\right) \rho_{c}^{\prime}\left(r_{c}^{\prime}\right) \\
& =-4 \pi \frac{G \rho_{c}^{\prime}\left(r_{c}^{\prime}\right)}{r_{c}^{\prime 2}} \int_{0}^{r_{c}^{\prime}} d \tilde{r} \tilde{r}^{2} \rho_{c}^{\prime}(\tilde{r}) .
\end{aligned}
$$

With the definition $\tau^{\prime} \equiv k_{B} T_{c}^{\prime} /\left(4 \pi m_{p} G\right)$, Equation (10) reduces to

$$
\tau^{\prime} \frac{d}{d r_{c}^{\prime}}\left(\frac{r_{c}^{\prime 2}}{\rho^{\prime}} \frac{d \rho^{\prime}}{d r_{c}^{\prime}}\right)=-\rho^{\prime} r_{c}^{\prime 2}
$$

The numerical solution to this nonlinear differential equation is plotted in Figure 2(a) for two values of $T_{c}^{\prime}$. As is also shown in that plot, the numerical solution is well approximated by

$$
n_{c}^{\prime}\left(r_{c}^{\prime}\right)=\frac{n_{0}^{\prime}}{1+\left(r_{c}^{\prime} / r_{0}^{\prime}\right)^{2}} .
$$

The normalization $n_{0}^{\prime}$ can be determined by integrating Equation (12) and equating it to Equation (5), and

$$
r_{0}^{\prime}=\sqrt{\frac{3 \tau^{\prime}}{m_{p} n_{0}^{\prime}}} .
$$

Naturally, the density drops to zero for $r_{c}^{\prime} \rightarrow \infty$. In order to make progress, we approximate the cloud as a sphere with outer boundary $R_{c}^{\prime}>r_{0}^{\prime}$ and set $n_{c}^{\prime}\left(r_{c}^{\prime} \geqslant R_{c}^{\prime}\right)=0$. Once the cloud hits the jet, it is ablated slice by slice beginning with a low particle-number region at the front, through the dense central region, and ending again at a low-density region at the rear end. Therefore, we define all quantities of the cloud as a function of $x^{\prime}$, the slice position with respect to the outer edge of the cloud that first touches the jet. That is, $x^{\prime}=0$ where the cloud first touches the jet, $x^{\prime}=R_{c}^{\prime}$ is the cloud's center, and $x^{\prime}=2 R_{c}^{\prime}$ marks the rear end of the cloud. With the speed of the cloud, it can be written as $x^{\prime}=v_{c}^{\prime} t^{\prime}$, where $t^{\prime}$ is the time that has passed since first contact in the AGN frame.

The number of particles ablated in each slice is the integral over the density $n_{c}^{\prime}\left(r_{c}^{\prime}\right)$ with respect to the slice volume. In the case of a sphere, the volume of a slice between positions $x^{\prime}$ and $x^{\prime}+d x^{\prime}$ is (Zacharias \& Schlickeiser 2013)

$$
d V_{s}^{\prime}\left(x^{\prime}\right)=d x^{\prime} \int d A_{s}^{\prime}\left(x^{\prime}\right)=\pi\left(2 R_{c}^{\prime} x^{\prime}-x^{\prime 2}\right) d x^{\prime},
$$

where $A_{s}^{\prime}(x)$ is the cross-section of a slice and $d x^{\prime}$ its width. The particle number in each slice then becomes

$$
d N_{s}^{\prime}\left(x^{\prime}\right)=d x^{\prime} \int n_{c}^{\prime}\left(r_{c}^{\prime}\right) d A_{s}^{\prime}\left(x^{\prime}\right)
$$

Writing the integral in cylindrical coordinates with $r_{c}^{\prime}\left(x^{\prime}\right)=\sqrt{\omega^{2}+\left(R_{c}^{\prime}-x^{\prime}\right)^{2}} \quad$ and $\quad \omega_{c}^{\prime}\left(x^{\prime}\right)=\sqrt{2 R_{c}^{\prime} x^{\prime}-x^{\prime 2}}$, Equation (15) becomes

$$
d N_{s}^{\prime}\left(x^{\prime}\right)=2 \pi d x^{\prime} \int_{0}^{\omega_{c}^{\prime}\left(x^{\prime}\right)} n_{c}^{\prime}\left(r_{c}^{\prime}(\omega)\right) \omega d \omega .
$$

Inserting Equation (12) into Equation (16), the integral can be easily solved, giving

$$
d N_{s}^{\prime}\left(x^{\prime}\right)=\pi d x^{\prime} r_{0}^{\prime 2} n_{0}^{\prime} \ln \left(\frac{r_{0}^{\prime 2}+R_{c}^{\prime 2}}{r_{0}^{\prime 2}+\left(R_{c}^{\prime}-x^{\prime}\right)^{2}}\right) .
$$

This function is shown in Figure 2(b) for two cases of $T_{c}$ along with an integration of the numerical solution of Equation (11). The analytical approximation and the exact result match nicely.

The injection of particles in the jet, which get dragged along and cause the flare at a shock somewhere downstream, can then be described by

$$
Q_{\mathrm{inj}}(t) \propto \ln \left(\frac{r_{0}^{\prime 2}+R_{c}^{\prime 2}}{r_{0}^{\prime 2}+\left(R_{c}^{\prime}-x^{\prime}\right)^{2}}\right) \delta\left(t-\frac{x^{\prime}}{v_{c}^{\prime}}\right) .
$$

Here, $\delta(q)$ is Dirac's $\delta$ function, which describes the slice-byslice ablation in time.

We stress that the entire mass of the cloud is not added to the jet at once, but gradually over about four months in the observer's frame. Hence, the impact of the added mass on the jet's bulk Lorentz factor at any given time is minor compared to the case where the entire cloud mass would be added at once. In the following, we assume a constant jet bulk Lorentz factor. 


\section{Modeling}

In order to model the long-term trend of the CTA 102 flare, we use the code by Diltz \& Böttcher (2014) and adapt it slightly to accommodate the variability induced by the cloud ablation as discussed in Section 3. The code calculates the electron distribution and photon emission spectra in the comoving frame of the emission region, and subsequently transforms it to the observer's frame taking into account the Doppler factor $\delta_{j}$, which we assume here to be equal to the bulk Lorentz factor $\Gamma_{j}$, and the redshift $z_{\text {red. }}$. The electron distribution function $n_{e}(\gamma, t)$ is calculated with a Fokker-Planck-type differential equation that takes into account injection, stochastic acceleration, cooling, and escape.

The injection electron distribution is of the form

$$
Q(\gamma, t)=Q_{0}(t) \gamma^{-s(t)} H\left[\gamma ; \gamma_{\min }(t), \gamma_{\max }(t)\right],
$$

where $\gamma$ is the electron Lorentz factor, $s$ the electron spectral index, and $H\left[\gamma ; \gamma_{\min }, \gamma_{\max }\right]$ denotes Heaviside's step function with $H=1$ for $\gamma_{\min } \leqslant \gamma \leqslant \gamma_{\max }$, and $H=0$ otherwise. The injection normalization is derived from input parameters as

$$
Q_{0}(t)=\frac{L_{j, e, \text { inj }}(t)}{V_{j} m_{e} c^{2}}\left\{\begin{array}{ll}
\frac{2-s(t)}{\gamma_{\max }^{2-s(t)}-\gamma_{\min }^{2-s(t)}} & \text { if } s \neq 2 \\
\left(\ln \frac{\gamma_{\max }}{\gamma_{\min }}\right)^{-1} & \text { if } s=2
\end{array},\right.
$$

with the electron injection luminosity $L_{j, e, \text { inj }}$ and the comoving volume $V_{j}=\frac{4}{3} \pi R_{j}^{3}$ of the emission region. Since the input parameters can be time dependent, the injection distribution might change in every time step.

The acceleration and escape terms are parameterized independent of energy. The escape timescale is defined by $t_{\text {esc }}=\eta_{\text {esc }} R / c$, namely, a multiple $\eta_{\text {esc }}$ of the light-crossing timescale. The acceleration timescale in turn is defined as a multiple $\eta_{\text {acc }}$ of the escape timescale: $t_{\text {acc }}=\eta_{\text {acc }} t_{\text {esc }}$.

The cooling term takes into account all radiative processes, namely synchrotron radiation in a randomly oriented magnetic field $B_{j}$, SSC, and IC emission on potential external fields, such as the accretion disk, the BLR, or a dusty torus. The IC process takes into account the full Klein-Nishina cross-section. The accretion disk spectrum is assumed to be of the Shakura \& Sunyaev (1973) type, which basically depends on the mass of the central black hole and the Eddington ratio $\eta_{\text {Edd }}$ of the disk luminosity $L_{\text {disk }}^{\prime}=\eta_{\text {Edd }} L_{\text {Edd }}^{\prime}$. The BLR spectrum is assumed to be a blackbody spectrum of effective temperature $T_{\mathrm{BLR}}^{\prime}$ normalized to the measured BLR luminosity. The size of the BLR is important to calculate the BLR energy density and potential absorption of $\gamma$-rays from the emission region. Similar definitions are possible for the dusty torus, but we ignore that photon field here due to a lack of observational evidence.

An implicit Crank-Nichelson scheme is used to solve the Fokker-Planck equation. With the solution for $n_{e}(\gamma, t)$, the radiation spectra are derived, which consider internal absorption through synchrotron self-absorption and external absorption of $\gamma$-rays through the external soft photon fields.

Before starting to model the light curve, we first derived two exemplary spectra of the low state before the flare in October and of the high state in late December in order to derive the baseline parameter sets that needed to be matched at these two states. The dates are MJD 57670 and MJD 57745, and are marked by the black and red vertical lines in the light curves of Figure 3, respectively. We chose these dates, since, in addition

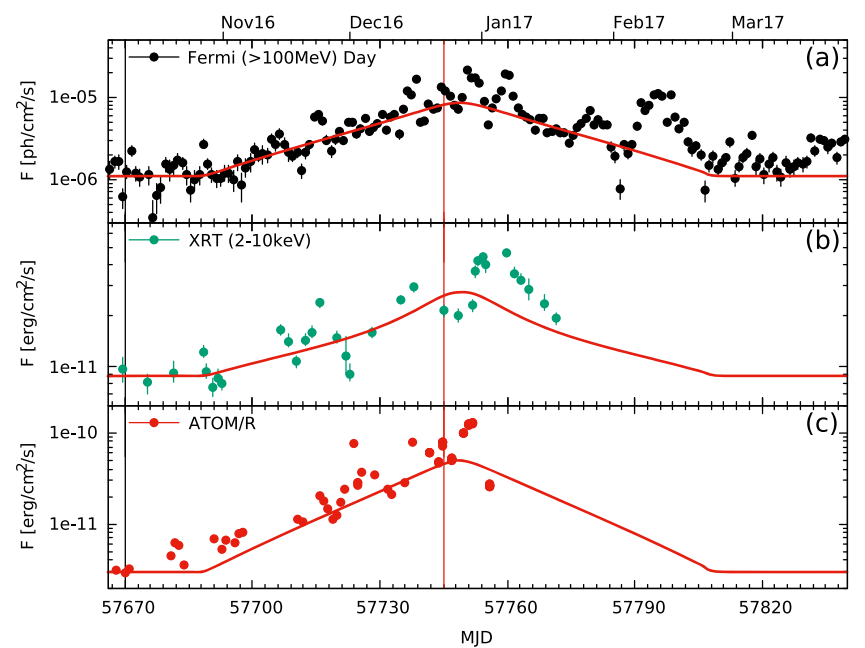

Figure 3. Light curves of the (a) Fermi-LAT data, (b) Swift-XRT data, and (c) ATOM/R data. The thick red lines are the modeling result, while the vertical thin black and red lines mark the dates when the spectra have been extracted. Note the logarithmic scaling of the $y$-axis.

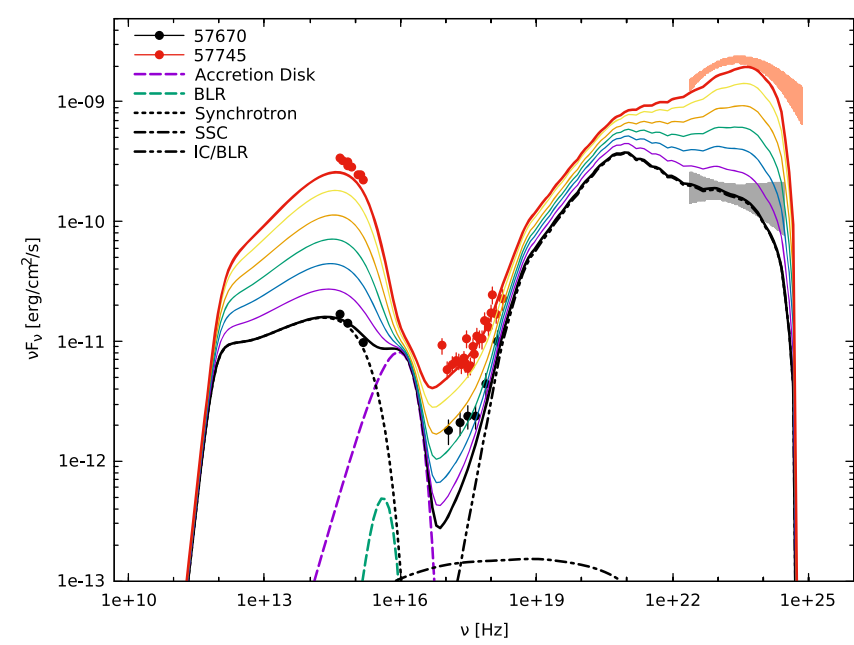

Figure 4. Two representative spectra of CTA 102 during the flare: MJD 57670 (black symbols and butterfly) and MJD 57745 (red symbols and butterfly). The Fermi-LAT spectra have been corrected for EBL absorption using the model of Franceschini et al. (2008). The thick black and red solid lines show model spectra for the beginning and the peak of the flare, while the thin solid lines (magenta, green, orange, yellow, blue) show the evolution of the model spectrum in roughly 10 day steps toward the maximum. The other lines give example curves of the composition of the spectrum: accretion disk (dashed magenta), BLR (dashed green), synchrotron (dotted black), SSC (dasheddotted black), and IC/BLR (dashed-double-dotted black).

to being representative of the respective flux levels, the data taken in all bands is contemporaneous. The spectra are shown in Figure 4. Most obvious are the significant flux changes between the two states and the change in peak energy of the IC component. The parameters of the fit to the low state are given in Table 1.

A few of these parameters are constrained by observations. The size of the emission region modulo the Doppler factor $R_{j} / \delta_{j}$ is constrained by the variability timescale in our data as $R_{j} \lesssim \Delta t^{\mathrm{obs}} c \delta_{j} /\left(1+z_{\text {red }}\right)$. Due to the measured optical intranight variability, the emission region must be smaller than a lightday in the observer's frame, corresponding to less than $4.5 \times 10^{16} \mathrm{~cm}$ for a Doppler factor of $\delta_{j}=35$. The chosen value of $R_{j}=2.5 \times 10^{16} \mathrm{~cm}$ is a compromise between the aforementioned limit and the necessity of a rather large 
Table 1

Model Parameter Description, Symbol, and Value

\begin{tabular}{lcc}
\hline \hline Definition & Symbol & Value \\
\hline Emission region distance to black hole & $z^{\prime}$ & $6.5 \times 10^{17} \mathrm{~cm}$ \\
Doppler factor of emission region & $\delta_{j}$ & 35 \\
Emission region radius & $R_{j}$ & $2.5 \times 10^{16} \mathrm{~cm}$ \\
Magnetic field of emission region & $B_{j}$ & $3.7 \mathrm{G}$ \\
Electron injection luminosity & $L_{j, e, \text { inj }}$ & $2.2 \times 10^{43} \mathrm{erg} \mathrm{s}^{-1}$ \\
Minimum electron Lorentz factor & $\gamma_{\min }$ & $1.3 \times 10^{1}$ \\
Maximum electron Lorentz factor & $\gamma_{\max }$ & $3.0 \times 10^{3}$ \\
Electron spectral index & $s$ & 2.4 \\
Escape time scaling & $\eta_{\mathrm{esc}}$ & 10.0 \\
Acceleration to escape time ratio & $\eta_{\mathrm{acc}}$ & 1.0 \\
Effective temperature of the BLR & $T_{\mathrm{BLR}}^{\prime}$ & $5.0 \times 10^{4} \mathrm{~K}$ \\
\hline Electron luminosity variation & $\Delta L_{j, e, \text { inj }}$ & $1.75 \times 10^{43} \mathrm{erg} \mathrm{s}$ \\
Electron spectral index variation & $\Delta s$ & -0.6 \\
Time between onset and peak of flare & $t_{f}^{\text {obs }}$ & 60 days \\
Cloud scale height & $r_{0}^{\prime}$ & $1.6 \times 10^{14} \mathrm{~cm}$ \\
\hline
\end{tabular}

Note. Values below the horizontal line mark parameters for the induced variability.

emission region in order to keep the SSC emission low. The latter would quickly overproduce the X-ray flux for smaller source radii especially during the variable period.

The magnetic field $B_{j}$ is constrained from the Compton dominance parameter $W$, which is defined as the ratio of the peak fluxes of the two spectral components. The peak fluxes are directly proportional to the underlying energy densities, namely the magnetic energy density and the energy density in the BLR photon field transformed to the comoving frame. Hence, $W=4 \Gamma_{j}^{2} u_{\mathrm{BLR}}^{\prime} / 3 u_{\mathrm{B}}$. Solving for $B_{j}$, one obtains

$$
\begin{aligned}
B_{j}= & \sqrt{\frac{8 \Gamma_{j}^{2} L_{\mathrm{BLR}}^{\prime}}{3 c R_{\mathrm{BLR}}^{\prime 2} W}} \\
= & 2.9\left(\frac{\Gamma_{j}}{10}\right)\left(\frac{W}{10}\right)^{-1 / 2}\left(\frac{L_{\mathrm{BLR}}^{\prime}}{4.14 \times 10^{45} \mathrm{erg} \mathrm{s}^{-1}}\right)^{1 / 2} \\
& \times\left(\frac{R_{\mathrm{BLR}}^{\prime}}{6.7 \times 10^{17} \mathrm{~cm}}\right)^{-1} \mathrm{G} .
\end{aligned}
$$

Unfortunately, the peak fluxes of both the synchrotron and the IC component are not well-defined in the low state. Hence, $W$ is not particularly well constrained, and values of at least 10 are plausible. We follow the standard assumption of the one-zone model that the magnetic field is tangled. Although this is a simplification, since one expects an ordered guide magnetic field in the jet, we have no observational constraints in hand that could constrain the geometry of the magnetic field during this particular event. Larionov et al. (2016) modeled their polarimetry data of the 2012 flare assuming a helical magnetic field and a helical motion of the emission region, which is different from our model.

The maximum electron Lorentz factor $\gamma_{\max }$ is constrained by the soft optical synchrotron spectrum. While a soft electron distribution could also account for the soft synchrotron spectrum, this would be inconsistent with the harder (than the optical spectrum) $\gamma$-ray spectrum. Hence, the soft optical spectrum can be interpreted as an exponential cutoff induced by a maximum electron Lorentz factor significantly below $10^{4}$. The minimum

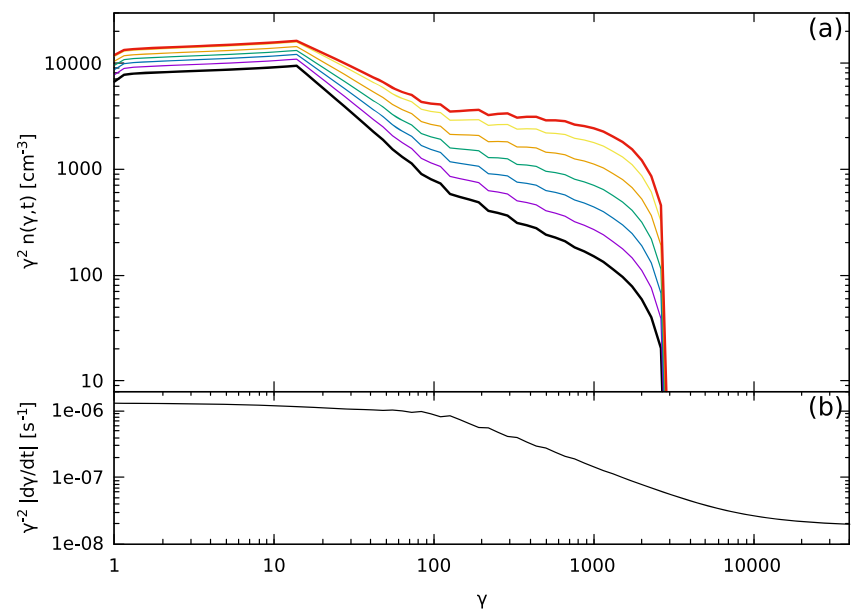

Figure 5. (a) Electron distribution function $\gamma^{2} n(\gamma, t)$ as a function of the electron Lorentz factor $\gamma$ for the same time steps as in Figure 4. (b) Electron cooling term $\gamma^{-2}|\dot{\gamma}|$ as a function of the electron Lorentz factor $\gamma$.

electron Lorentz factor $\gamma_{\min }$ is not constrained by the observations, but has been chosen in such a way that the IC/BLR spectrum fits well the hard X-ray spectrum. In principle, the $\gamma$-ray spectrum could be used to constrain the spectral index $s$. However, as one can see in Figure 5, the Klein-Nishina effect in the cooling changes the particle spectrum considerably at the particle energies that correspond to the $\gamma$-ray spectrum probed by Fermi-LAT (see also the discussion below). Hence, the standard relations between photon spectra and (un)cooled particle distributions do not work, and we chose the spectral index to match well the Fermi-LAT spectrum.

The observed luminosity of $L^{\mathrm{obs}} \sim 10^{48} \mathrm{erg} \mathrm{s}^{-1}$ of the ground state is at the high end of FSRQ luminosities (e.g., Ghisellini et al. 1998). In order to reduce the required particle energy densities, we chose a relatively high Doppler factor of $\delta_{j}=35$. However, observations of the MOJAVE program revealed radio knots moving with apparent speeds of $\sim 18 c$ (Lister et al. 2016), which permit Doppler and Lorentz factors in the chosen order of magnitude.

Although there is no observational constraint on the distance of the emission region from the black hole $z^{\prime}$, it is chosen in such a way that the emission region is immersed in BLR photons, but still the attenuation of $\gamma$-rays by the BLR photons is minimal. Closer to the black hole, the attenuation would start to become important even in the HE domain, resulting in softer spectra and a much poorer fit. A greater distance from the black hole would result in an inefficient IC/BLR process. Hence, the emission region should be located around the outer edge of the BLR.

The results are insensitive to the escape time scaling $\eta_{\mathrm{esc}}$ and the acceleration to escape time ratio $\eta_{\text {acc }}$, since the strong cooling (see Figure 5) dominates over the escape and acceleration for all energies. The effective temperature of the BLR $T_{\mathrm{BLR}}^{\prime}$ is also not constrained by observations, but it impacts the onset of the Klein-Nishina domain in the IC process. The chosen value implies that the Klein-Nishina domain already sets in for electron Lorentz factors of $\sim 100$. Lower values of $T_{\mathrm{BLR}}^{\prime}$ would increase the electron turnover energy slightly.

We note that the chosen parameter set is not unique, and other parameter sets might give equivalent results. However, 
the precise parameters are not important for the evolution of the event, which is the main concern of this paper.

In order to model the evolution of the flare, we varied the electron injection luminosity following Equation (18) as

$$
L_{j, e, \text { inj }}(t)=L_{j, e, \text { inj }}+\Delta L_{j, e, \text { inj }} \ln \left(\frac{t_{0}^{2}+t_{f}^{2}}{t_{0}^{2}+\left(t_{f}-t\right)^{2}}\right)
$$

where all parameters are considered in the comoving frame, implying $t_{f}=\delta_{j} t_{f}^{\text {obs }} /\left(1+z_{\text {red }}\right)$, and the timescale $t_{0}=\delta_{j} v_{c}^{\prime} r_{0}^{\prime}$ is related to the cloud's scale height $r_{0}^{\prime}$. There is no observational constraint on the latter value. We know that the cloud's scale height $r_{0}^{\prime}$ must be smaller than the cloud's radius $R_{c}^{\prime}$. We tested a few values and found that the value related to the scale height given in Table 1 gives the best fit. A larger scale height than the one used underpredicts the fluxes, while a smaller scale height produces a narrow peak, which is also inconsistent with the observations.

In order to account for the changing peak energy of the IC component, we also change the electron spectral index. Due to a lack of constraints, we assume a linear change as

$$
s(t)=s+\Delta s \frac{t_{f}-\left|t_{f}-t\right|}{t_{f}} .
$$

With $\Delta s$ being negative (see Table 1), the injection spectrum hardens until the maximum of the flare and subsequently returns to the pre-flare value. We further assume that the bulk Lorentz factor of the emission region is constant.

The resulting model spectra are shown in Figure 4. The fit of the pre-flare and high-state spectra (black and red curves) is quite good, taking into account that we do not aim for a precise fit. The colored spectra show the evolution of the spectrum from the low state toward the maximum in roughly 10 day intervals. The lack of evidence of a broken power-law spectrum in the X-ray domain gives us confidence that the seemingly poor fit at low X-ray energies is not a big concern. The upturn of the model curves around $100 \mathrm{MeV}$ is due to a change in the cooling behavior at these and higher energies, as shown in Figure 5(b). At low energies, the cooling is dominated by the IC/BLR process, but reduces for electron Lorentz factors $\gamma>100$ due to the Klein-Nishina effect. This hardens the electron distribution, as can be seen in Figure 5(a), where we show the underlying particle distribution of each photon spectrum of Figure 4. For electron Lorentz factors $\gamma>10^{4}$, synchrotron cooling becomes dominant, which is however unimportant for the present study, since we do not consider electrons with these energies. Since neither the BLR nor the magnetic field is assumed to vary, the electron cooling term is constant in time. The small wiggles in the $\gamma$-ray spectra, the particle distributions, and the cooling term are due to numerical inaccuracies.

The resulting model light curves are shown as thick red lines in Figure 3. We present the light curves with a logarithmic $y$-axis in order to highlight the significant change in flux and the details of the theoretical light-curve evolution. We model the $\gamma$-ray, X-ray, and optical $R$ band. We ignore the other optical/UV bands, since the $R$ band is the most detailed synchrotron light curve, and the constant color implies that the behavior in the other bands is very similar. The model of the general evolution of the flare is very good in all energy bands.

\section{Discussion}

The modeling gives a good representation of the overall flare profile. We can safely conclude that the long-term activity of CTA 102 is consistent with the addition of a large amount of mass to the jet over a time period of a few months. We modeled this by the penetration of the jet by a gas cloud, for which we only made the assumption of being in hydrostatic equilibrium. Below, we will discuss the potential origin of the cloud.

Given that we use the IC/BLR process to model the highenergy component of the spectrum, the cloud-jet interaction should take place within the BLR. We set the emission region close to the outer edge of the BLR, allowing the IC/BLR process to operate, while the absorption at $\gamma$-rays is kept low. Hence, the cloud could originate from the BLR itself.

From the above modeling, we can deduce that the electron density at the beginning of the flare is $n_{j, e, \text { min }}=2.32 \times$ $10^{4} \mathrm{~cm}^{-3}$, which rises to $n_{j, e, \text { max }}=4.0 \times 10^{4} \mathrm{~cm}^{-3}$ at the peak of the flare. With Equation (5), $a=0.1$, and the parameters given in Table 1, we can calculate the number of particles in the cloud to be $N_{c}^{\prime}=2.34 \times 10^{54}$ or a mass of $M_{c}=$ $N_{c}^{\prime} m_{p}=3.9 \times 10^{30} \mathrm{~g} \sim 0.1 \% M_{\odot}$. The speed of the cloud is, Equation (3), $v_{c}^{\prime}=5.12 \times 10^{8} \mathrm{~cm} \mathrm{~s}^{-1}$, and its radius, Equation (4), $R_{c}^{\prime}=1.3 \times 10^{15} \mathrm{~cm}$. The average particle density in the cloud is, thus, $n_{c}^{\prime}=2.54 \times 10^{8} \mathrm{~cm}^{-3}$. The scale height of the cloud is $r_{0}^{\prime}=1.6 \times 10^{14} \mathrm{~cm}$. This value, along with Equation (5), Equation (13), and an integration of Equation (12), implies a temperature

$$
\begin{aligned}
T_{c}^{\prime} & =\frac{G m_{p}^{2} N_{c}^{\prime}}{6 k_{B} r_{0}^{\prime}\left[\left(R_{c}^{\prime} / r_{0}^{\prime}\right)-\arctan \left(R_{c}^{\prime} / r_{0}^{\prime}\right)\right]} \\
& \sim 0.5 \mathrm{~K} .
\end{aligned}
$$

This temperature is clearly too low, since a gas cloud cannot become colder than the cosmic microwave background. Additionally, standard parameters for BLR clouds suggest a radius of $\sim 10^{13} \mathrm{~cm}$ and an average density of $10^{9-11} \mathrm{~cm}^{-3}$ (Dietrich et al. 1999; Peterson 2006). Hence, the size of our model cloud is too large, while the density is too low. However, all of these parameters were derived under the assumption that the entire cloud is devoured by the jet, and this does not include potential higher density regions responsible for the fast but bright flares on top of the long-term trend. These higher density regions would exhibit higher temperatures, likely raising the temperature of the entire cloud. Additionally, the collision of the cloud with the jet might induce a shock wave running through the former (e.g., Poludnenko et al. 2002; Araudo et al. 2009), which could lead to the ejection of cloud material away from the interaction site. Then, our estimate is only a lower limit on the matter content of the cloud, and the particle number, and hence the temperature, could be significantly higher. Furthermore, we assumed that the hydrostatic equilibrium is solely mediated by an isothermal gas. If the cloud contains a significant magnetic field, it will stabilize the cloud even if the temperature is exceeding the isothermal temperature derived above.

Although these considerations could lead to a density and temperature of the cloud that more closely resemble parameters of BLR clouds, it does not influence our estimate of the size $R_{c}^{\prime}$, which solely depends on the speed of the cloud. Since we assumed Keplerian motion of the cloud, the size of the cloud depends inversely on the square root of the distance from the black hole. Hence, the size of the cloud can be reduced, if the 
ablation takes place farther away from the black hole. Although this could bring the size closer to the BLR cloud parameters, a BLR cloud can be excluded, since our model is already placed close to the outer edge of the BLR. In such a case, the highenergy component cannot be due to IC/BLR, and more likely hadronic scenarios need to be invoked. Assuming that a shock at parsec-scale distance from the black hole can efficiently accelerate protons, the flare could be proton induced, since the cloud provides the jet with the same amount of protons as electrons. We will elaborate on a hadronic scenario for the flare elsewhere.

The constraint on the maximum electron Lorentz factor is particularly strong from the shape of the synchrotron spectrum. Hence, the cutoff of the IC component is fixed at $\sim 20 \mathrm{GeV}$, which does not even take into account absorption by the EBL. If the spectrum of CTA 102 is indeed mainly shaped by the leptonic model as described here, CTA 102 cannot be detected at very high-energy $\gamma$-rays $(E>100 \mathrm{GeV})$ by ground-based Cherenkov experiments.

In summary, we showed that the prolonged and strong activity of the FSRQ CTA 102 could have been caused by the full or partial ablation of a gas cloud colliding with the jet. From the assumption of hydrostatic equilibrium of an isothermal gas with the gravity of the cloud, we derived the density structure of the cloud. This structure is reflected in the injection of material ablated by the jet causing the severalmonth-long outburst. Our model light curves are in good agreement with the observations. The model parameters suggest that the cloud was not fully ablated, and much of the material might have been lost during the collision.

The authors are grateful for fruitful discussions with Heike Prokoph, Moritz Hackstein, and Chris Diltz, as well as for a constructive report by the anonymous referee.

The work of M.Z. and M.B. is supported through the South African Research Chair Initiative (SARChI) of the South African Department of Science and Technology (DST) and National Research Foundation. ${ }^{7}$ F.J. and S.J.W. acknowledge support by the German Ministry for Education and Research (BMBF) through Verbundforschung Astroteilchenphysik grant 05A11VH2. J.-P.L. gratefully acknowledges CC-IN2P3 (cc. in2p3.fr) for providing a significant amount of the computing resources and services needed for this work. A.W. is supported by the Foundation for Polish Science (FNP).

\section{ORCID iDs}

M. Zacharias (iD https://orcid.org/0000-0001-5801-3945

M. Böttcher (1) https://orcid.org/0000-0002-8434-5692

J.-P. Lenain (ib https://orcid.org/0000-0001-7284-9220

A. Wierzcholska (i) https://orcid.org/0000-0003-4472-7204

\section{References}

Acero, F., Ackermann, M., Ajello, M., et al. 2015, ApJS, 218, 23

Acero, F., Ackermann, M., Ajello, M., et al. 2016, ApJS, 223, 26

Araudo, A. T., Bosch-Ramon, V., \& Romero, G. E. 2009, A\&A, 503, 673

Araudo, A. T., Bosch-Ramon, V., \& Romero, G. E. 2010, A\&A, 522, A97

Arnaud, K. A. 1996, in ASP Conf. Ser. 101, Astronomical Data Analysis Software and Systems V, ed. G. H. Jacoby \& J. Barnes (San Francisco, CA: ASP), 17

Atwood, W. B., Abdo, A. A., Ackermann, M., et al. 2009, ApJ, 697, 1071

Bachev, R., Popov, V., Strigachev, A., Semkov, E., et al. 2017, MNRAS, 417, 2216

Blandford, R., \& Königl, A. 1979, ApL, 20, 15

Blandford, R., \& Rees, M. J. 1974, MNRAS, 169, 395

Bosch-Ramon, V. 2015, A\&A, 575, A109

Bosch-Ramon, V., Perucho, M., \& Barkov, M. V. 2012, A\&A, 539, A69

Burrows, D. N., Hill, J. E., Nousek, J. A., \& Kennea, J. A. 2005, SSRv, 120,165

de la Cita, V. M., del Palacio, S., Bosch-Ramnon, V., et al. 2017, A\&A, 604, A39

Dietrich, M., Wagner, S. J., Courvoisier, T. J.-L., Bock, H., \& North, P. 1999, A\&A, 351, 31

Diltz, C., \& Böttcher, M. 2014, JHEAp, 1, 63

Franceschini, A., Rodighiero, G., \& Vaccari, M. 2008, A\&A, 487, 837

Fromm, C. M., Perucho, M., Ros, E., et al. 2011, A\&A, 531, A95

Gehrels, N., Chincarini, G., Giommi, P., et al. 2004, ApJ, 611, 1005

Ghisellini, G., Celotti, A., Fossati, G., Maraschi, L., \& Comastri, A. 1998, MNRAS, 301, 45

Giommi, P., Blustin, A. J., Capalbi, M., et al. 2006, A\&A, 456, 911

Hauser, M., Möllenhoff, C., Pühlhofer, G., et al. 2004, AN, 325, 659

Kalberla, P. M. W., Burton, W. B., Hartmann, D., et al. 2005, A\&A, 440, 775

Klein, R. I., McKee, C. F., \& Colella, P. 1994, ApJ, 420, 213

Komissarov, S. S. 1994, MNRAS, 269, 394

Larionov, V. M., Villata, M., Raiteri, C. M., et al. 2016, MNRAS, 461, 3047

Lister, M. L., Aller, M. F., Aller, H. D., et al. 2016, AJ, 152, 12

Mattox, J. R., Bertsch, D. L., Chiang, J., et al. 1996, ApJ, 461, 396

Perucho, M., Bosch-Ramon, V., \& Barkov, M. V. 2017, A\&A, 606, A40

Perucho, M., Marti, J. M., Laing, R. A., \& Hardee, P. E. 2014, MNRAS, 441, 1488

Peterson, B. M. 2006, LNP, 693, 77

Pian, E., Falomo, R., \& Treves, A. 2005, MNRAS, 361, 919

Poludnenko, A. Y., Frank, A., \& Blackman, E. G. 2002, ApJ, 576, 832

Schlafly, E. F., \& Finkbeiner, D. P. 2011, ApJ, 737, 103

Schramm, K.-J., Borgeest, U., Camenzind, M., et al. 1993, A\&A, 278, 391

Shakura, N. I., \& Sunyaev, R. A. 1973, A\&A, 24, 337

Zacharias, M., \& Schlickeiser, R. 2013, ApJ, 777, 109

Zamaninasab, M., Clausen-Brown, E., Savolainen, T., \& Tchekhovskoy, A. 2014, Natur, 510, 126

\footnotetext{
7 Any opinion, finding and conclusion, or recommendation expressed in this material is that of the authors, and the NRF does not accept any liability in this regard.
} 